\title{
Infrared interferometry of circumstellar envelopes
}

\author{
John D. Monnier \\ Physics Dept, University of California, Berkeley, CA 94720-7450, USA
}

\begin{abstract}
This paper will review the technical progress of interferometric infrared observing techniques from the first 2-element interferometer 25 years ago to the $3+$ element arrays now coming into service. To date, only the Infrared Spatial Interferometer (ISI) has published separateelement interferometric data on circumstellar dust shells in the infrared and many of these scientific results will be discussed. Speckle interferometry has also evolved significantly over the last few decades as slit-scanning techniques over single-pixel detectors have largely been replaced by fastreadout of large format detector arrays. Important near-infrared and mid-infrared results derived from speckle data will also be reviewed.

Until recently, two-dimensional information about circumstellar dust distributions has been sorely lacking, hence detections of dust shell asymmetries have been difficult and uncertain. New speckle observations using modern, 10-m class telescopes have yielded surprising results, demonstrating the importance of accurate closure phase information in interpreting interferometric data. These discoveries hopefully precursor those to be made from closure-phase imaging with the new generation of separateelement, interferometric arrays.
\end{abstract}

\section{Introduction}

Infrared interferometry arises from the desire to image the heavens in ever increasing spatial resolution. Other techniques exist with the same goal in mind, but the limited space afforded to this review necessarily restricts the scope to exclude lunar occultation (e.g., Ragland, Chandrasekhar \& Ashok 1997; Käufl et al. 1998) and adaptive optics works (e.g., Cruzalèbes et al. 1998; Monnier et al. 1999). This review will also ignore the rapidly increasing interferometric successes at observing the stellar surfaces themselves (Dyck et al. 1996; Wilson, Dhillon \& Haniff 1997; Burns et al. 1998; Van Belle et al. 1998; Tuthill, Monnier \& Danchi 1999; Young et al. 1999), but rather will concentrate on the circumstellar envelopes of late-type stars.

The objects under scrutiny here are mass-losing asymptotic giant branch (AGB) stars; many of these stars are Mira variables, both with oxygen- and carbon-rich shells. This volume is devoted to the study of these objects and I invite you to look through the other review articles in order to gain a greater understanding of these interesting astrophysical laboratories. That said, what do we hope to measure with interferometers and then hopefully will learn about the astrophysics of the AGB? Interferometrists strive to get the most detailed 
information possible concerning the morphologies of circumstellar dust shells. This requires observations at various wavelengths as well as at various times. These measurements then can be used to directly test theories of dust production and mass-loss mechanisms in general, which are important in guiding our understanding of stellar evolution along the AGB. When coupled with spectral line observations, high angular resolution studies can directly measure gas densities, velocities, and temperatures around these stars, as well as probe the chemistry of molecular formation.

\section{Introduction to interferometry}

Although images, or maps, can be made from interferometry data (a process known as aperture synthesis), an interferometer actually measures a quantity called the visibility. When light beams from two separate telescopes are brought together to produce a fringe signal, the visibility is proportional to the strength of the detected fringes. The visibility, which is a function of the projected telescope separation (or baseline), can be shown to be equivalent to one of the Fourier component amplitudes of the object brightness distribution. Nice discussions of interferometry can be found in Thompson, Moran \& Swenson (1986) and Born \& Wolf (1964).

For reconstructing reliable images of complex extended objects, one also requires accurate estimates of the Fourier phases, not just the amplitudes. While atmospheric turbulence scrambles the phase of individual Fourier components, a quantity called the closure phase retains its integrity. By summing the fringe phases for baselines in a closed triangle, the closure phase is formed and allows a majority of the intrinsic phase information to be recovered for interferometric arrays with three or more telescopes (e.g., Jennison 1958; Readhead et al. 1988). This quantity is critically important for accurate reconstructions of highly asymmetric structures in images.

Since any image can be alternatively represented by its Fourier components, the collection of all "interesting" components allows the interferometric data to be inverted, thus producing an image. The Caltech VLBI and the VLBMEM packages can be used to estimate the original object brightness distribution using Fourier inversion algorithms such as CLEAN (with self-calibration) and the Maximum Entropy Method (e.g., Högbom 1974; Cornwell \& Wilkinson 1981; Sivia 1987; Haniff et al. 1989). Speckle interferometry data (more on this later) is often reduced using a bispectral analysis code which performs a direct inversion from the estimated Fourier amplitudes and phases. However, modifications of this technique lead to more robust reconstructions in the presence of noise (e.g., Hofman \& Weigelt 1993).

\section{First generation of infrared Michelson interferometers}

The first interferometric observations of circumstellar dust shells were performed by the Arizona group (McCarthy \& Low 1975). The interferometer setup was mounted at the Cassegrain focus of a large telescope, beams from two subapertures interfering together onto a single-pixel InSb detector (later upgraded to a Ge bolometer). Additional observations were presented in McCarthy, Low \& 
Howell (1977), McCarthy, Howell \& Low (1978, 1980), and McCarthy (1979). These measurements were sensitive in the mid-infrared, usually between $5 \mu \mathrm{m}$ and $12.5 \mu \mathrm{m}$.

Meanwhile C. H. Townes was leading the U.C. Berkeley group in building a separate-element heterodyne interferometer. Infrared light incident on each of the Auxiliary McMath Solar Telescopes at Kitt Peak (5.5 m separation) were combined with a $\mathrm{CO}_{2}$ laser operating near $10 \mu \mathrm{m}$ to produce a down-converted $\mathrm{RF}$ signal from a $\mathrm{Ge}: \mathrm{Cu}$ detector (later $\mathrm{HgCdTe}$ ). The $\mathrm{RF}$ signals (original bandwidth $\approx \pm 1200 \mathrm{MHz}$ ) from the two telescopes were sent through a delay line and subsequently correlated, acting much like a typical radio or $\mathrm{mm}$ interferometer. This system detected fringes on the limb of Mercury as early as 1974 (Johnson 1975), and observations of circumstellar envelopes soon followed (Sutton et al. 1977, 1978, 1979).

These two groups observed many of the same stars and reached the same general conclusions. A diversity of dust shell types were detected, both large and small (in terms of stellar size). Both groups measured changes in the dust shell around the Mira variable $o$ Cet, and attributed these to the large amplitude pulsation. These first set of measurements also indicated asymmetries for some of the thickest (i.e. most resolved) dust shells (McCarthy 1979).

\section{One-dimensional speckle interferometry}

The promise of speckle interferometry was first realized by Antoine Labeyrie (Labeyrie 1970). In the original formulation, short exposures of an astrophysical object are made to freeze the "speckling" induced by the turbulent atmosphere. The amount of high-resolution structure in the speckle pattern, as quantified by its power spectrum, is a measure of two things: 1) the quality of the atmospheric seeing, and 2) the high resolution structure in the object of interest. Observing a nearby point-source star allows the calibration of the seeing contribution and thus the extraction of interferometric visibility measurements out to the diffraction limit of the telescope. It was only later that the Fourier phases were estimated from such data (e.g., Knox \& Thompson 1974; Weigelt 1977).

Although speckle interferometry was applied to measurements of visible star diameters and binary star orbits in the early- to mid-1970's, it was not until 1979 that the first infrared application to AGB circumstellar envelopes emerged. A group from France published a number of papers on the subject, resolving a few dust shells for the first time in the near-infrared (Foy et al. 1979; Sibille, Chelli \& Léna 1979; Mariotti et al. 1983). Because of the lack of two-dimensional array cameras, a single pixel detector (typically $\mathrm{InSb}$ ) was used to sample the speckle cloud. In most setups, a slit was scanned across the speckled image (or the image was passed over the slit). Power spectra for all scans were averaged together to estimate the visibility, after calibrating the mean atmospheric (and telescope) transfer function.

While many groups made speckle observations in the 1980's, I want to discuss the few representing significant advances. Dyck et al. (1984) contains the largest sample of resolved dust shells to date, observing 16 late-type stars and proto-planetary nebula (PPN) between $1.65 \mu \mathrm{m}$ and $4.8 \mu \mathrm{m}$. This work was able to confirm, in a general sense, that dust condenses at a few stellar radii from 
the stellar surface, and the existence of dust shell asymmetries for a number of the most resolved sources.

Another significant advance occurring was the use of radiative transfer models for interpreting visibility curves. Because spatial resolution is often inadequate to unambiguously interpret the data, radiative transfer codes allow the inner radius of dust formation to be estimated and can utilize multi-wavelength data in a natural way. Two illustrations can be found in Ridgway et al. (1986) and Ridgway \& Keady (1988), which estimated the dust shell optical depths for IRC +10216 , NML Cyg, and IRC +10420 .

A number of other firsts occurred during this very productive time. Dyck, Beckwith \& Zuckerman (1983) published the first (and only?) infrared speckle observations on spectral lines, five fundamental vibration-rotation lines of $\mathrm{CO}$ around IRC +10216 . Phase retrieval methods were first applied to reconstructing 1-D profiles of asymmetric dust shells (Chelli, Perrier \& Biraud 1983; Leinert \& Haas 1989; Navarro, Benitez \& Fuentes 1990), and speckle techniques were applied in the mid-infrared (Cobb \& Fix 1987; Benson, Turner \& Dyck 1989). Dyck et al. (1991) compared the existing IRC +10216 speckle data to show that the circumstellar emission changed significantly during the 1980's. There were a number of other interesting near-infrared (e.g., Dainty et al. 1985; Dyck et al. 1987; Mariotti et al. 1992; Lopez et al. 1993) and mid-infrared speckle results (Fix \& Cobb 1988; Dyck \& Benson 1992) published during this time.

\section{The Infrared Spatial Interferometer}

After the successful interferometry experiment at Kitt Peak, the UC Berkeley group began constructing a pair of movable telescopes to be placed on $\mathrm{Mt}$. Wilson, California, based on the same heterodyne detection design (present bandwidth $\approx \pm 2700 \mathrm{MHz}$ ). The Infrared Spatial Interferometer (ISI) published its first results on IRC +10216 in 1990 (Danchi et al. 1990; Bester et al. 1991) and showed the dust shell size changes as a function of the stellar pulsational phase.

The most significant single paper was a compilation of dust shell sizes for 13 late-type stars (Danchi et al. 1994), which established two classes of dusty stars, those with hot dust close to the star and those with detached, relatively cool shells. In addition, the variation of inner radii indicated an episodic emission time-scale of 20-50 years. The ISI has monitored a number of AGB stars for a 10-year period now, discovering complex shell geometries and time variation around multiple sources (Greenhill et al. 1995; Bester et al. 1996; Monnier et al. 1997; Hale et al. 1997; Lopez et al. 1997). High spatial resolution observations with $\lambda / \Delta \lambda \approx 10^{5}$ presently underway should reveal the location of $\mathrm{NH}_{3}$ and $\mathrm{SiH}_{4}$ molecules, and help explain their anomalously high observed abundances around AGB stars (e.g., Betz, McLaren \& Spears 1979; Goldhaber 1988).

\section{Two-dimensional speckle interferometry}

Two major technical advances in the 1990's have ushered in a renaissance in speckle observations. The development of fast-readout, infrared array cameras allows the entire speckle cloud to be recorded and thousands of images to be 
collected in a short period of time. Secondly, a new generation of large aperture telescopes has come into service, allowing 2-3 times higher spatial resolution.

Most present results are coming from two groups using different techniques and telescopes, although other groups are making significant contributions as well (e.g., Haniff \& Buscher 1998; Buscher, Haniff \& Oudmaijer 1998). A team headed by G. Weigelt has been using so-called "speckle masking," a modified bispectral analysis, to analyze speckle data collected at the $6 \mathrm{~m}$ Special Astrophysical Observatory telescope and the 3.5m+ ESO telescopes on La Silla, Chile. Very nice results have recently been reported (Osterbart et al. 1997; Weigelt et al. 1998; Osterbart et al. 1998; Irrgang et al. 1998; Weigelt et al. 1999). P. Tuthill leads the other group, which uses aperture masking techniques on the Keck-I telescope with maximum baselines of $11 \mathrm{~m}$. Results can be found in this volume (Tuthill et al. 1999) and in other publications (Tuthill, Monnier \& Danchi 1998; Monnier et al. 1999).

The aperture masking experiment at Keck has succeeded in obtaining good visibility and Fourier phase information down to K-band magnitude of 5. Probably, standard speckle techniques will need to be used to go much dimmer. Using "speckle masking" (not to be confused with "aperture masking"), Weigelt's group has been able to go down to $\mathrm{K} \sim 9$.

Both groups have succeeded in constructing very detailed images at the telescope diffraction limit. The resolution achieved equals about one stellar radius for the closest objects (e.g., IRC +10216 ), allowing the dust formation process to be studied in detail. Three different groups presented images of IRC +10216 at $2.2 \mu \mathrm{m}$, and all images showed the same asymmetric and clumpy dust shell (Weigelt et al. 1998; Haniff \& Buscher 1998; Tuthill et al. 1999). In addition, proper motions of the dust clouds have been detected, which promises to yield important dynamical and three-dimensional information on the dust shell geometry. These observations are showing that mass-loss processes can be very inhomogeneous in the inner dust shell regions, for reasons not entirely clear.

\section{Conclusions}

I have summarized the progress of infrared interferometry on circumstellar envelopes in Fig. 1 and Table 1. Fig. 1 shows the spatial resolution and wavelength coverage attainable with present instruments, while Table 1 lists all dust shells resolved by papers referenced here (and is a fairly complete list of published results). This list will surely grow by a dozen or so in the coming years just from analyses of existing data, especially from the speckle imaging groups. While many measurements do exist, a lack of coordinated observations at multiple wavelengths has hindered interpretation of these data because of the time variable nature of these sources. Also, combining speckle observations with polarization and spectral line work can make unique and valuable contributions to the study of AGB stars, but have only rarely been performed. In addition, photometric monitoring of these sources in the infrared can be very valuable when trying to understand temporal changes in the dust shell properties, but is also rarely done, despite their high flux levels.

The inhomogeneous, asymmetric and time-variable dust shells observed around many stars indicate that mass-loss on the AGB may not be understood 


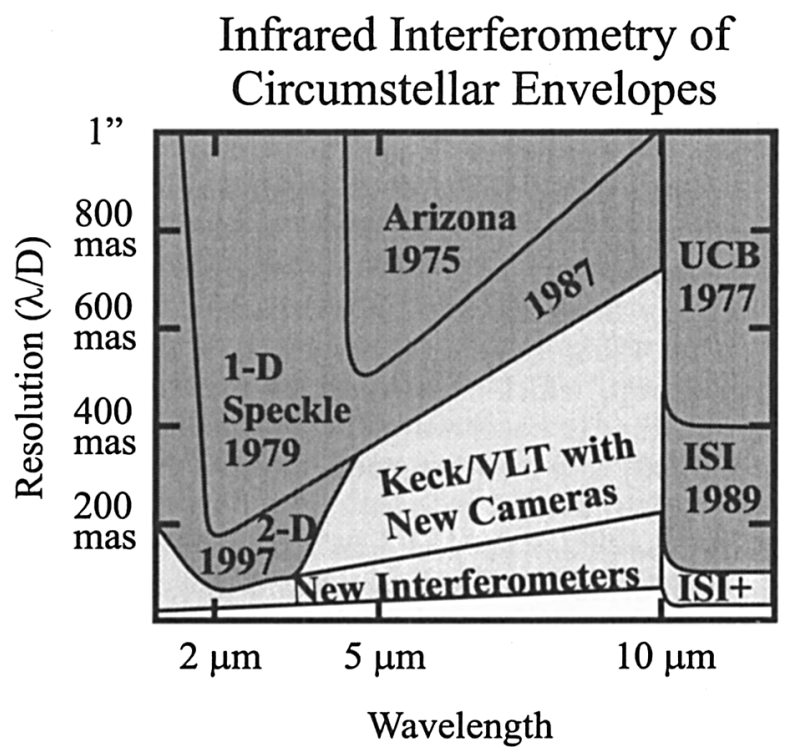

Figure 1. Spatial resolution vs. wavelength for past, present and future interferometric observations. Parameter space to be covered in the next few years is shaded in light grey.

quite as well as previously thought. More theoretical work and modeling (twoor even three-dimensional radiative transfer) are needed to make full use of the new high resolution data. Understanding of these processes should continue to improve as a number of infrared interferometer arrays are being constructed or upgraded (IOTA, COAST, PTI, CHARA, Keck, VLTI, MIRA, ISI+, more), leading to spatial resolutions (at some wavelengths) of less than 1 mas in the near future.

Acknowledgments. I am thankful to A. P. Zemgulys for enlightening discussions.

\section{References}

Benson J.A., Turner N.H., Dyck H.M., 1989, ApJ 97, 1763

Bester M., Danchi W.C., Degiacomi C.G., Townes C.H., Geballe T.R., 1991, ApJ 367, L27

Bester M., Danchi W.C., Hale D., et al., 1996, ApJ 463, 336

Betz A.L., McLaren R.A., Spears D.L., 1979, ApJ 229, 97

Born M., Wolf E., 1964, Principles of Optics, Macmillan, New York

Burns D., et al., 1998, MNRAS 298, 697

Buscher D.F., Haniff C.A., Oudmaijer R.D., 1998, poster contribution, this conference 
Table 1. Circumstellar shells resolved by infrared interferometry

\begin{tabular}{|c|c|c|c|}
\hline Star name & Type & Wavelengths $(\mu \mathrm{m})$ & Other comments \\
\hline NML Cyg & Red Supergiant & $\begin{array}{c}2.2,2.4,3.5 \\
3.6,3.8,4.8 \\
5.0,7.9,8.7,10 \\
10.4,11.4,12.6\end{array}$ & Asymmetric \\
\hline$\alpha$ Ori & Red Supergiant & $\begin{array}{c}7.9,8.7,10,10.2 \\
10.4,11.1,11.4\end{array}$ & Time variable \\
\hline VY CMa & Red Supergiant & $\begin{array}{c}1.65,2.2,3.1 \\
3.8,4.8,8.3 \\
10.2,11.1\end{array}$ & $\begin{array}{l}\text { Asymmetric } \\
2 \text {-D images }\end{array}$ \\
\hline S Per & Red Supergiant & $\begin{array}{c}7.9,8.7,10 \\
10.4,11.4,12.6\end{array}$ & \\
\hline $\begin{array}{l}\alpha \text { Sco } \\
\text { VX Sgr }\end{array}$ & Red Supergiant & $10.0,11.1$ & Asymmetric, 1-D profile \\
\hline $\begin{array}{l}\text { VX Sgr } \\
\mu \text { Cep }\end{array}$ & $\begin{array}{l}\text { Red Supergiant } \\
\text { Red Supergiant }\end{array}$ & $\begin{array}{c}2.2,3.8,11.15 \\
10\end{array}$ & \\
\hline$\alpha$ Her & Red Supergiant & 11.15 & \\
\hline $\mathrm{IRC}+10216$ & Carbon Star & $\begin{array}{c}2.2,3.1,3.5 \\
8.3,10,10.2 \\
11.1,12.5\end{array}$ & $\begin{array}{l}\text { Asymmetric, Time variable, } \\
\text { Polarization data, 1-D profiles, } \\
\text { Spectral Line Work (CO, } \mathrm{NH}_{3}, \\
\left.\mathrm{SiH}_{4}\right), 2 \text {-D images, } \\
\text { Proper motions detected }\end{array}$ \\
\hline CIT 6 & Carbon Star & $\begin{array}{l}2.2,3.1,3.8 \\
4.8,10,11.5\end{array}$ & $\begin{array}{l}\text { Asymmetric, } \\
2 \text {-D images }\end{array}$ \\
\hline IRAS $15194-5115$ & Carbon Star & $2.2,3.6$ & \\
\hline CIT 5 & Carbon Star & 4.8 & \\
\hline$\chi$ Cyg & Mira & $\begin{array}{l}2.2,4.6,4.8 \\
10,11.15\end{array}$ & Asymmetric \\
\hline IK Tau & Mira & $3.8,11.15$ & Time variable \\
\hline$o$ Cet & Mira & 10.2 & Asymmetric, Time Variable \\
\hline R Leo & Mira & 11.1 & \\
\hline $\mathrm{R}$ Cas & Mira & $\begin{array}{c}7.9,8.7,10 \\
10.4,11.4,12.6\end{array}$ & \\
\hline CIT 3 & Mira & $1.65,2.2,11.15$ & Asymmetric \\
\hline AFGL 2290 & Mira & 2.2 & Asymmetric \\
\hline T Cas & Mira & 10 & \\
\hline U Her & Mira & 10 & \\
\hline U Ori & Mira & 11.15 & \\
\hline W Aql & Mira & 11.15 & \\
\hline R Aqr & Mira & 11.15 & \\
\hline BC Cyg & Mira? & $\begin{array}{c}7.9,8.7,10 \\
10.4,11.4,12.6\end{array}$ & \\
\hline BI Cyg & Mira? & $\begin{array}{c}7.9,8.7,10 \\
10.4,11.4,12.6\end{array}$ & \\
\hline RX Boo & Semiregular & $\begin{array}{c}7.9,8.7,10 \\
10.4,11.4,12.6\end{array}$ & \\
\hline $\begin{array}{l}\text { SW Vir } \\
\text { X Her }\end{array}$ & Semiregular & $2.4,10$ & \\
\hline $\begin{array}{l}\text { X Her } \\
\text { OH } 26.5+0.6\end{array}$ & $\begin{array}{c}\text { Semiregular } \\
\text { OH/IR }\end{array}$ & $\begin{array}{c}10 \\
3.8,4.6,4.8 \\
5,10\end{array}$ & Asymmetric \\
\hline OH 354.9-0.5 & $\mathrm{OH} / \mathrm{IR}$ & 10 & Asymmetric \\
\hline $\mathrm{OH} 1.1-0.8$ & $\mathrm{OH} / \mathrm{IR}$ & 10 & Asymmetric \\
\hline Red Rectangle & PPN & $\begin{array}{c}1.25,1.6,2.2 \\
3.1,3.3,3.8 \\
4.8\end{array}$ & $\begin{array}{l}\text { Asymmetric, 1-D Profile, } \\
\text { Polarization data, } \\
\text { 2-D images }\end{array}$ \\
\hline $\mathrm{OH} 231.8+4.2$ & PPN & 3.8 & Asymmetric \\
\hline AFGL 618 & PPN & 3.8 & \\
\hline M1-9 & PPN & $3.8,4.8$ & Asymmetric \\
\hline M2-9 & PPN & 3.8 & Asymmetric \\
\hline IRC +10420 & F8-G0 I & $\begin{array}{l}2.2,3.5,3.8 \\
4.8,5,10\end{array}$ & Asymmetric \\
\hline$\eta \mathrm{Car}$ & LBV & 4.6 & 1-D profiles, 2-D images \\
\hline
\end{tabular}


Chelli A., Perrier C., Biraud Y.G., 1983, A\&A 117, 199

Cobb M.L., Fix J.D., 1987, ApJ 315, 325

Cornwell T.J., Wilkinson P.N., 1981, MNRAS 196, 1067

Cruzalebes P., Lopez B., Bester M., Gendron E., Sams B., 1998, A\&A 338, 132

Dainty J.D., Pipher J.L, Lacasse M.G., Ridgway S.T., 1985, ApJ 293, 530

Danchi W.C., Bester M., Degiacomi C.G., Greenhill L.J., Townes C.H., 1994, AJ 107, 1469

Danchi W.C., Bester M., Degiacomi C.G., McCullough P.R., Townes C.H., 1990, ApJ 359, 59

Dyck H.M., Beckwith S., Zuckerman B., 1983, ApJ 271, L79

Dyck H.M., Zuckerman B., Leinert Ch., Beckwith S., 1984, ApJ 287, 801

Dyck H.M., Zuckerman B., Howell R.R., Beckwith S., 1987, PASP 99, 99

Dyck H.M., Benson J.A., Howell R.R., Joyce R.R., Leinert C., 1991, AJ 102, 200

Dyck H.M., Benson J.A., 1992, AJ 104, 377

Dyck H.M., Benson J.A., Van Belle G.T., Ridgway S.T., 1996, AJ 111, 1705

Fix J.D., Cobb M.L., 1988, ApJ 329, 290

Foy R., Chelli A., Sibille F., Léna P., 1979, A\&A 79, L5

Goldhaber D.M., 1988, University of California at Berkeley, PhD Dissertation

Greenhill L.J., Colomer F., Moran J.M., et al., 1995, ApJ 449, 365

Hale D.D.S., Bester M., Danchi W.C., et al., 1997, ApJ 490, 407

Haniff C.A., Buscher D.F., Christou J.C., Ridgway S.T., 1989, MNRAS 241, 51

Haniff C.A., Buscher D.F., 1998, A\&A 334, L5

Hofman K.-H., Weigelt G., 1993, A\&A 278, 328

Högbom J., 1974, ApJS 15, 417

Irrgang P., Balega Y.Y., Gauger A., Osterbart R., Schniggenberg G., Weigelt

G., 1998, poster contribution, this conference

Jennison R.C., 1958, MNRAS 118, 276

Johnson M.A., 1975, University of California at Berkeley, PhD Dissertation

Käufl H.U., Stecklum B., Richichi A., Richter S., 1998, poster contribution, this conference

Knox K.T., Thompson B.J., 1974, ApJ 193, L45

Labeyrie A., 1970, A\&A 6, 85

Leinert C., Haas M., 1989, A\&A 221, 110

Lopez B., Perrier C., Mekarnia D., Lefèvre J., Gay J., 1993, A\&A 270, 462

Lopez B., Danchi W.C., Bester M., et al., 1997, ApJ 488, 807

Mariotti J.-M., Chelli A., Foy R., et al., 1983, A\&A 120, 237

Mariotti J.-M., Monin J.-L., Ghez P., Perrier C., Zadrozny A., 1992, A\&A 255, 462

McCarthy D.W., Low F.J., 1975, ApJ 202, L37

McCarthy D.W., Low F.J., Howell R., 1977, ApJ 214, L85

McCarthy D.W., Howell R., Low F.J., 1978, ApJ 223, L113 
McCarthy D.W., 1979, in High Angular Resolution Stellar Interferometry, IAU Colloq. 50, p. 18

McCarthy D.W., Howell R., Low F.J., 1980, ApJ 235, L27

Monnier J.D., Tuthill P.G., Lopez B., et al., 1999, ApJ 512, in press

Monnier J.D., Bester M., Danchi W.C., et al., 1997, ApJ 481, 420

Navarro R., Benitez D., Fuentes F.J., 1990, J. Opt. Soc. Am. A 7, 459

Osterbart R., Langer N., Weigelt G., 1997, A\&A 325, 609

Osterbart R., Balega Y.Y., Langer N., Man'shchikov A.B., Weigelt G., 1998, poster contribution, this conference

Ragland S., Chandrasekhar T., Ashok N.M., 1997, MNRAS 287, 681

Readhead A.C.S., Nakajima T.S., Pearson T.J., Neugebauer G., Oke J.B., Sargent W.L.W., 1988, AJ 95, 1278

Ridgway S.T., Joyce R.R., Connors D., Pipher J.L., Dainty C., 1986, ApJ 302, 662

Ridgway S.T., Keady J.J., 1988, ApJ 326, 843

Sibille F., Chelli A., Léna P., 1979, A\&A 79, 315

Sivia D.S., 1987, Cambridge University, PhD Dissertation

Sutton E.C., Betz A.L., Storey J.W.V., Spears D.L., 1979, ApJ 230, L105

Sutton E.C., Storey J.W.V., Betz A.L., Townes C.H., Spears D.L., 1977, ApJ 217, L97

Sutton E.C., Storey J.W.V., Townes C.H., Spears D.L., 1978, ApJ 224, L123

Thompson R., Moran J.M., Swenson G.W., 1986, Interferometry and Aperture Synthesis in Radio Astronomy, John Wiley \& Sons

Tuthill P.G., Monnier J.D., Danchi W.C., 1998, A Half Century of Stellar Pulsation Interpretations, p. 322

Tuthill P.G., Monnier J.D., Danchi W.C., 1999, this volume

Van Belle G.T., PTI Collaboration, Thompson R., 1998, poster contribution, this conference

Weigelt G., 1977, Opt. Commun. 21, 55

Weigelt G., Balega Y., Blöcker T., Fleischer A.J., Osterbart R., Winters J.M., 1998, A\&A 333, L51

Weigelt G., Balega Y.Y., Blöcker T., Fleischer A.J., Hofmann K.-H., Osterbart R., Winters J.M., 1999, this volume

Wilson P.W., Dhillon V.S., Haniff C.A., 1997, MNRAS 291, 819

Young J.S., et al., 1999, this volume 


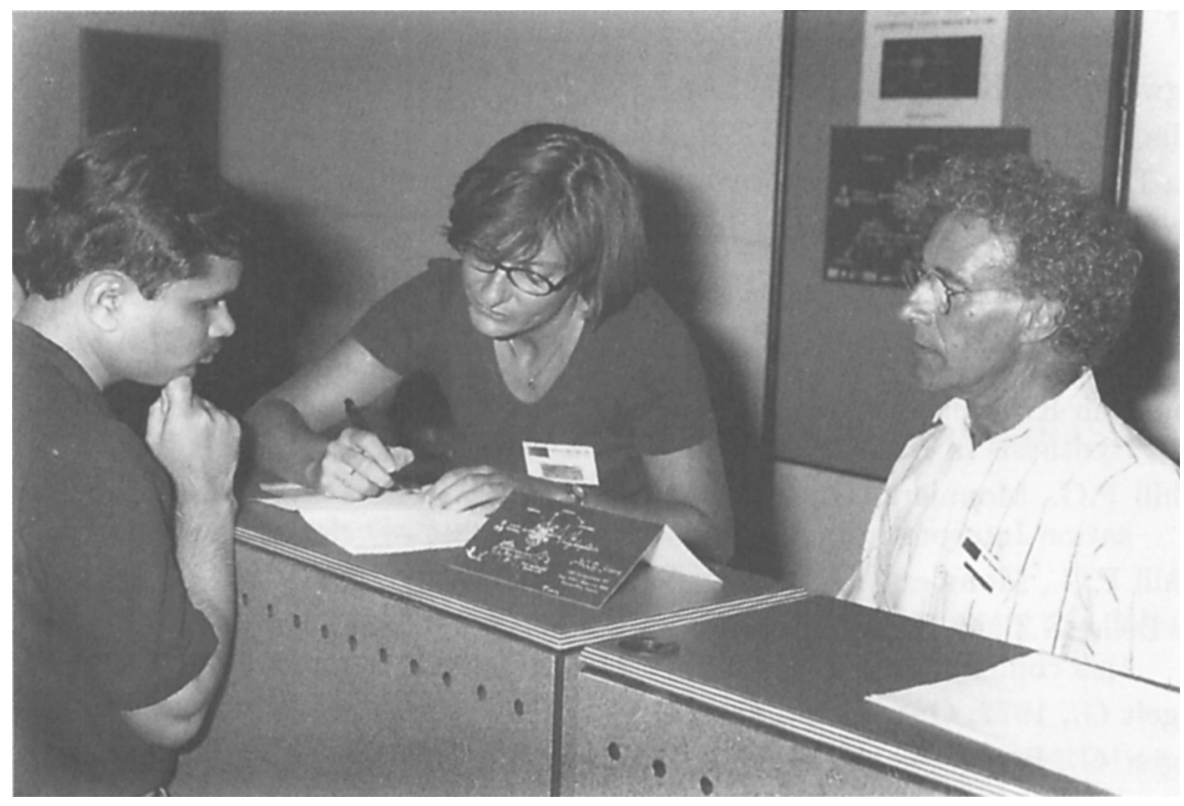

Busy L.O.C. (Agnès Lèbre with Christian Magnan) and Devendra Ojha 